\title{
Evolución del gasto en alimentos procesados en la población del Gran Santiago en los últimos 20 años
}

\author{
MIRTA CROVETTO ${ }^{1, a}$, RICARDO UAUY²
}

\section{Changes in processed food expenditure in the population of Metropolitan Santiago in the last twenty years}

Background: During recent decades household incomes have increased steadily, leading to changes in dietary habits and food expenditure. Aim: To report changes in household food expenditure focusing on trends in processed food across income quintiles in Metropolitan Santiago. Material and Methods: Information obtained from the Fourth (1986-1987), Fifth (1996-1997) and Sixth National Institute of Statistics (INE) Surveys (2006-2007) were used. Food expenditure data over the study periods was extracted from household expenditure surveys (HES) after verifying and adjusting food prices registered by the INE to current 2007 prices. Results: Absolute food expenditure over the study period increased for all groups; the largest increase was found in lowest income quintiles; however, the proportion of total family budget spent on food decreased in all groups. The largest increases in food expenditure corresponded to sweetened beverages, processed fruit juices, alcoholic drinks, ready meals and "eating out". Expenditure on fish rose slightly whereas the absolute spending on legumes, eggs and oils decreased. Expenditure in processed food as percentage of total food expenditure increased from $42 \%$ to $57 \%$ of total for mean household. For the $2^{\text {nd }}$ quintile it rose from $31 \%$ to $48 \%$ and for the lowest quintile increased from $53 \%$ to 68\% over the three decades (1987-2007). Changes were greatest in energy rich processed foods such as bread, pastries, confectioneries and granulated sugar. These particular foods are of high energy density and contain high amounts of saturated fat, sodium and added sugars. Conclusions: These results confirm that food consumption patterns over the past decades have progressively departed from the recommended dietary guidelines given by national and international health organizations.

(Rev Med Chile 2012; 140: 305-312).

Key words: Foods, specialized; Food, preserved; Nutrition processes; Chile.
${ }^{1}$ Facultad de Ciencias de la Salud, Universidad de Playa Ancha, Valparaíso, Chile. ${ }^{2}$ Instituto de Nutrición y Tecnología de los Alimentos, Universidad de Chile, Santiago; Chile. ${ }^{a}$ Nutricionista, Msc.

Recibido el 14 de junio de 2011, aceptado el 21 de noviembre de 2011.

Correspondencia a: Mirta Crovetto M. Avenida Playa Ancha No 270

Playa Ancha, Valparaíso Fax: (56-32) 2286713 E-mail: mcrovetto@upla.cl
$\mathrm{E}$ n las tres últimas décadas el poder adquisitivo de los hogares ha aumentado, modificando los patrones de consumo de la población del Gran Santiago. Estudios realizados sobre las tres últimas encuestas de presupuestos familiares (EPF); 1986-1987 (IV); 1996-1997 (V); 2006-2007 (VI), ilustran los cambios en el patrón alimentario entre 1987 y $1997^{1-3}$. Todos los quintiles de ingreso aumentan el consumo aparente de alimentos procesados (alta densidad energética), grasas saturadas, sodio, azúcares agregados y pier- den importancia las verduras, frutas y legumbres, (alimentos naturales, fuentes de fibra dietaria y fitoquímicos protectores) y productos del mar, (fuente de ácidos grasos omega-3; eicosapentaenoico (EPA) y docosahexanoico DHA) ${ }^{4,6}$.

Los cambios han sido catalizados, entre otros factores, por la incorporación de tecnologías en la elaboración de alimentos que facilitan su preparación y consumo y, de estrategias de marketing que inducen a preferirlos. La evolución de las diferencias en el consumo alimentario se 
asocia en el tiempo con los notables cambios en la prevalencia de enfermedades crónicas como las cardiovasculares y el cáncer. Estos antecedentes nos llevaron a examinar las variaciones en el consumo aparente de alimentos, distinguiendo patrones de consumo más y menos compatible con la salud basados en las recomendaciones de la Organización Mundial de la Salud (OMS) del año $2003^{7}$. Esta diferenciación se caracteriza por el grado y procesamiento de los alimentos y su potencial impacto en la salud. En este contexto, la clasificación recientemente propuesta por Monteiro y cols, que clasifica a los alimentos en mínimamente procesados, medianamente procesados y ultraprocesados, y el reciente artículo de Ludwig et al, establecen el rol del procesamiento de los alimentos en la causalidad de las enfermedades crónicas relacionadas con la nutrición ${ }^{8,9}$. Alimentos mínimanente procesados, son aquellos, que en el proceso industrial las propiedades saludables de los alimentos no se modifican sustancialmente y su objetivo es facilitar su consumo. Se envasan sin adición de componentes que afecten la salud, como el sodio en exceso. Se incluyen carne, leche, granos enteros, legumbres, alimentos frescos y perecederos sin procesar; leguminosas, nueces, frutas, hortalizas, raíces y tubérculos. En los medianamente procesados, se combinan procesos como; prensado, trituración, molienda, refinación, "purificar», hidrogenación, hidrólisis, extrusión, uso de enzimas y aditivos, obteniendo productos manufacturados o ingredientes culinarios (aceites, grasas, azúcar y edulcorantes, almidones, el jarabe de maíz alto en fructosa, lactosa, leche y proteínas de soja, las gomas, conservantes y aditivos de cosméticos y otros). En general, aportan energía. Los ultraprocesados, son combinaciones de los anteriores para la obtención de productos casi listos, de fácil y rápida preparación. Son altos en grasas totales, saturadas o trans, azúcares, sodio, carentes de micronutrientes, fibra dietética y de alta densidad energética9,10.

El objetivo general de este estudio fue describir el patrón alimentario actual y los cambios en el consumo aparente de alimentos, basado en las tendencias del gasto absoluto y relativo en alimentos de los hogares en los últimos 20 años; comparando la VI (2006-07) con la V (1996-07) y la IVEPF (1986-07) de los hogares del Gran Santiago; caracterizar los cambios en el gasto $y$, en especial en los alimentos procesados y su potencial efecto sobre la salud y orientar acciones que promuevan un patrón de consumo más saludable.

Este artículo, analizará el gasto en alimentación de los hogares para el total de hogares (TH), quintil II (QII) y quintil V (QV), representativos del promedio de la población y de los sectores de menores y mayores ingresos. En lo sucesivo, nos referiremos a cada período, indicando el año 1987; 1997 y 2007, respectivamente.

\section{Material y Métodos}

Se utilizó la información contenida en la IV, V y VI EPFs, realizadas por el Instituto Nacional de Estadísticas (INE) ${ }^{1-3}$. Los datos están ordenados por ingreso per cápita y la metodología se basó en el análisis comparativo del gasto por hogar en alimentos para el total de hogares y quintiles de ingreso, en base a los precios registrados por el INE actualizados a abril de $2007^{11-13}$. El gasto en alimentos se refiere sólo al que realizan los hogares en forma privada según su capacidad de compra. La metodología ha sido descrita por los autores en publicaciones anteriores ${ }^{4-6}$. En este artículo, se agregan aspectos principales de la metodología, relacionada con la última encuesta analizada. a) Se ordenó y homologó la información de la IV EPF, en base a las 131 agrupaciones de la VI y VI EPF. b) Se mantuvo la clasificación y ordenamiento de los16 grupos de alimentos; c) Para la homologación del gasto en alimentos de las tres encuestas a pesos de abril 2007, se aplicó un factor de actualización con un deflactor calculado en base al Índice de Precios de los Alimentos (IPA) de junio de 1988, enero de 1997 y abril de 2007; d) Se mantuvo la metodología en la clasificación y ordenamiento de las comidas preparadas para llevar al hogar y las comidas fuera del hogar; e) La clasificación en no procesados y procesados se basó en los criterios definidos previamente. Se consideraron no procesados o naturales al pan, hortalizas, frutas frescas, secas, desecadas, legumbres, leche, huevos, carnes y pescados sin procesos de transformación incorporados. Los otros productos, incluyendo a las comidas preparadas para llevar y fuera del hogar, se consideraron procesados. Los antecedentes epidemiológicos analizados corresponden a los resultados de la Encuesta Calidad de Vida (ECV) 2000, 2006; Encuesta Nacional de Salud (ENSA) 2003 y $2009-2010^{14-17}$. 
Se analizó la información del gasto en alimentos proveniente de la IV (19861987) y V EPF, (1997-1998) (3,4 $^{3,4}$ estudios nacionales publicados previamente sobre el perfil epidemiológico ${ }^{1,2}$; Encuesta Calidad de Vida 2000, 2006; Encuesta Nacional de Salud (ENS) 2003 y otras fuentes de información $\mathrm{n}^{5-8}$.

El ordenamiento de los datos obtenidos en las EPFs, se realizó por medio del programa Microsoft Office Excel 2003.

\section{Resultados}

Evolución del gasto absoluto y relativo en alimentos de los hogares del Gran Santiago

El gasto absoluto en alimentos, se refiere al gasto en dinero que realizan los hogares (Tabla 1). El gasto en alimentos entre 1987-2007 tuvo un aumento progresivo de $52,9 \% ; 75,9 \%$ y de $42,4 \%$ para el TH; QII, y QV, respectivamente. Entre 1987-1997, se observa el mayor incremento, en especial en los quintiles de menores ingresos e ingresos medios de $41,7 \%$; $57,2 \%$ y $34,7 \%$, para el TH; QII y QV, simultáneamente. El gasto relativo, definido como la ponderación del gasto de cada producto respecto al gasto total en alimentos, disminuyó en el período, representando 21\%, $31,2 \%$ y $14,5 \%$ para el TH, QII y QV, respectivamente, en el 2007 (Figura 1).

\section{Cambios del gasto absoluto en alimentos según grupos} de alimentos

Entre 1987-2007, se observa un aumento en el gasto absoluto en la mayoría de los grupos de alimentos, a excepción de huevos, legumbres, aceites y grasas (Figura 2). El gasto en pescados y mariscos tiene un leve incremento y representa

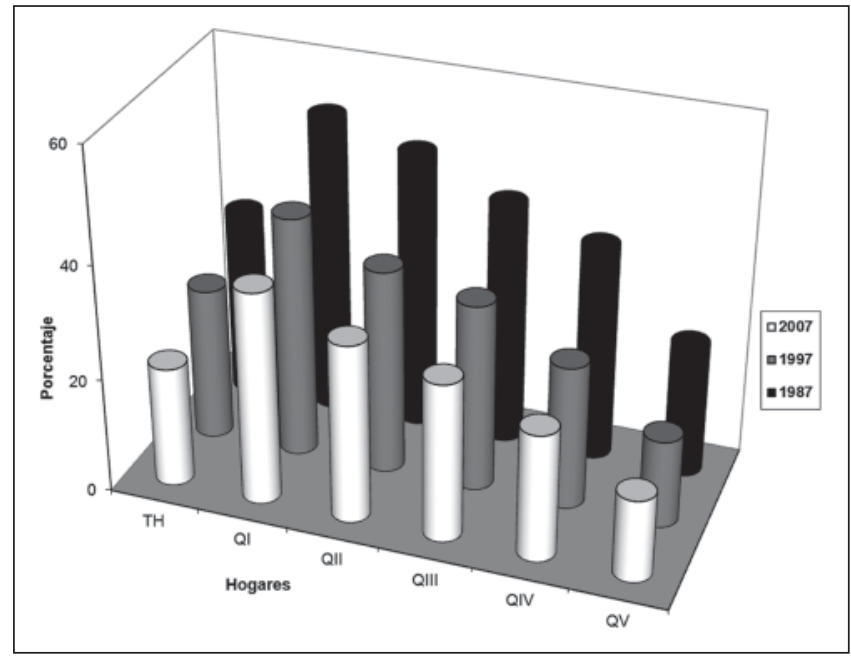

Figura 1. Evolución Gasto Relativo en Alimentos. Total Hogares y Quintiles de Ingreso. Gran Santiago*. 1987-1997-2007. En porcentaje (\%) ${ }^{*}$ ) En pesos abril de 2007. Deflactado por IPA.

Tabla 1. Evolución del gasto absoluto y relativo en alimentos. Total hogares y quintiles de Ingreso. Gran Santiago. 1987-1997-2007. En pesos $(*)$ y porcentaje (\%)

\begin{tabular}{|c|c|c|c|c|c|c|c|c|c|c|c|c|}
\hline & TH & $\%$ & QI & $\%$ & QII & $\%$ & QIII & $\%$ & QIV & $\%$ & QV & $\%$ \\
\hline $\begin{array}{l}\text { Gasto absoluto y } \\
\text { relativo } 1987\end{array}$ & 105.119 & 32,9 & 54.146 & 52,3 & 70.449 & 48,4 & 91.320 & 42.3 & 119.987 & 37,6 & 187.878 & 23,1 \\
\hline $\begin{array}{l}\text { Gasto absoluto y } \\
\text { relativo1997 }\end{array}$ & 148.961 & 26,8 & 75.973 & 42,3 & 110.961 & 35,9 & 135.572 & 32.9 & 168.177 & 25,2 & 252.963 & 15,4 \\
\hline $\begin{array}{l}\text { Gasto absoluto y } \\
\text { relativo } 2007\end{array}$ & 160.782 & 21,1 & 87.424 & 37,4 & 123.944 & 31,4 & 146.747 & 27.9 & 178.293 & 22,4 & 267.502 & 14,5 \\
\hline $\begin{array}{l}\text { Porcentaje varia- } \\
\text { ción 1986-07 }\end{array}$ & 41,7 & & 40,3 & & 57,5 & & 48,5 & & 40,2 & & 34,6 & \\
\hline $\begin{array}{l}\text { Porcentaje varia- } \\
\text { ción 1996-07 }\end{array}$ & 7,9 & & 11,5 & & 11,7 & & 8,2 & & 6,0 & & 5,7 & \\
\hline $\begin{array}{l}\text { Porcentaje varia- } \\
\text { ción 2006-07 }\end{array}$ & 52,9 & & 61,5 & & 75,9 & & 60,7 & & 48,6 & & 42,4 & \\
\hline
\end{tabular}

(*) En pesos abril 2007. Deflactado por IPA. 


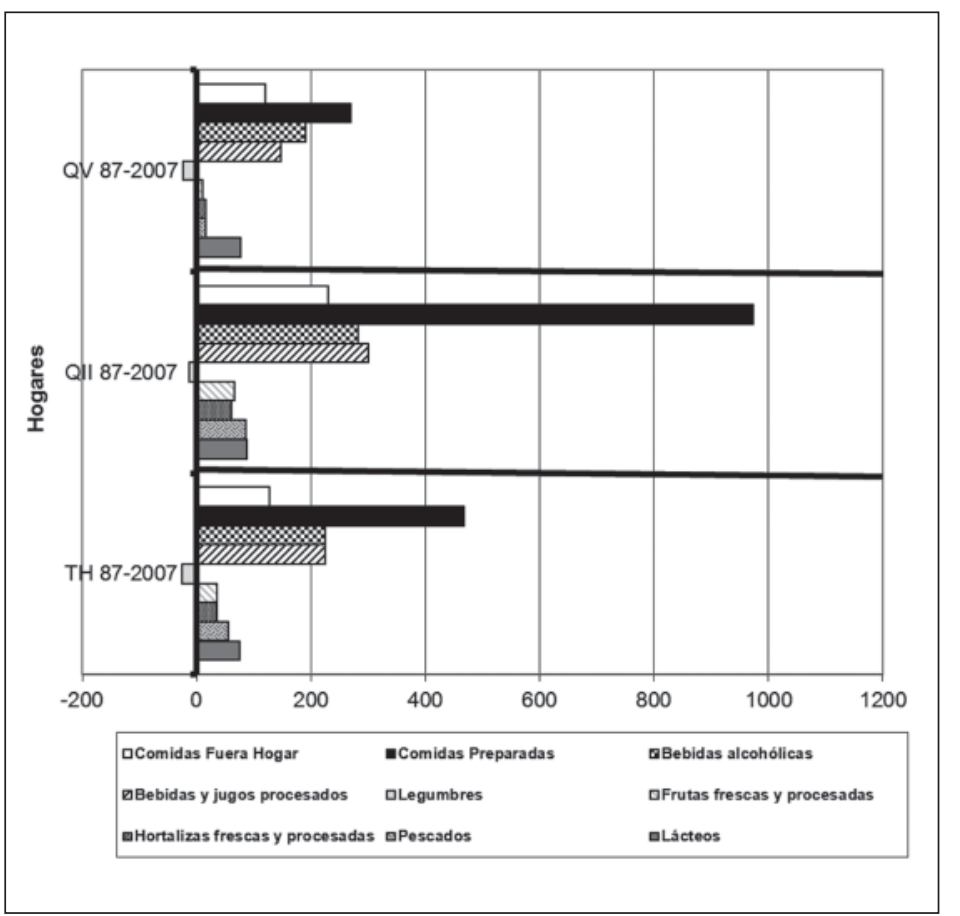

Figura 2. Cambios en el gasto absoluto por grupos de alimentos. Total Hogares y Quintiles de Ingreso II y V. Gran Santiago*. 1987-2007. En porcentaje (\%). (*) En pesos abril 2007. Deflactado por IPA.

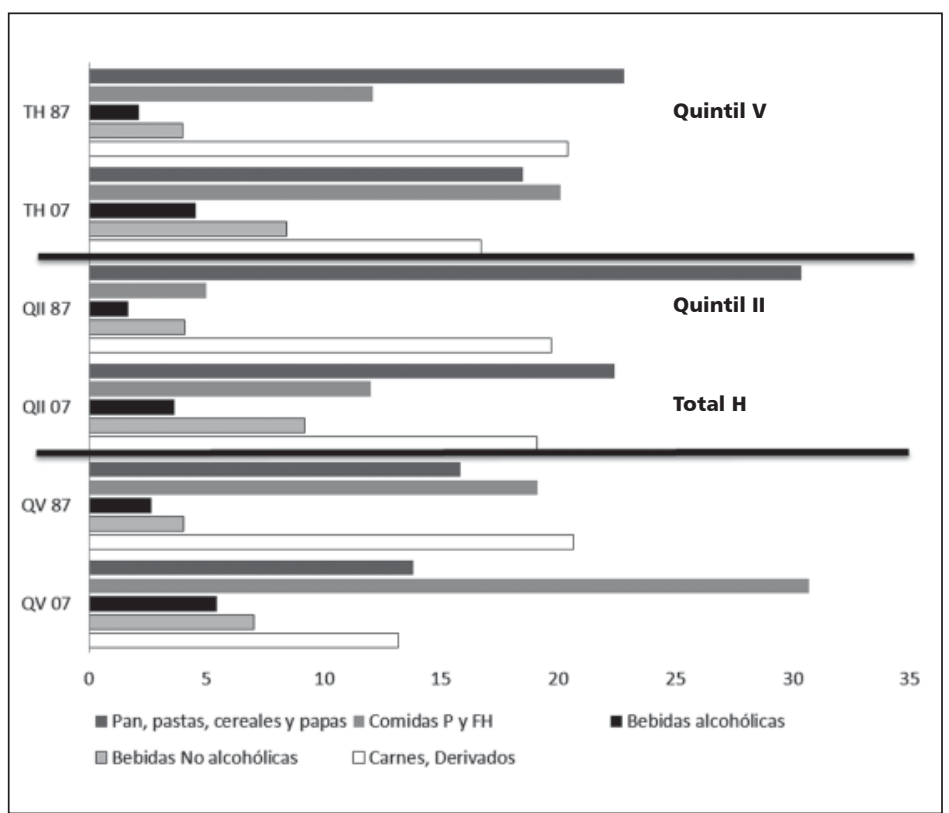

Figura 3. Gasto relativo gasto según grupo de alimentos respecto al gasto total. Total Hogares y Quintiles de Ingreso II y V*. Gran Santiago 1987-2007. En porcentaje (\%). (*) En pesos abril 2007. Deflactado por IPA. menos de $10 \%$ del gasto carnes y derivados procesados del $\mathrm{TH}$ y QII. En legumbres, se mantiene la tendencia decreciente observada desde 1987, el gasto declarado, indica que los hogares no alcanzan a gastar en un kilo de legumbres al mes. En hortalizas y frutas hay un aumento para todos los hogares, destacando el QII con un aumento de $61 \%$ y $67 \%$ respectivamente. A pesar de ello, el gasto en este ítem es inferior al de bebidas, jugos procesados y bebidas alcohólicas, situación que se repite en lácteos, para el TH y QII, el que es menor al de bebidas y jugos procesados. Destaca el aumento en la participación del gasto absoluto en bebidas, jugos procesados, bebidas alcohólicas, comidas preparadas y fuera del hogar para todos los grupos quintiles.

\section{Gasto relativo (Tabla 2)}

Se observa el descenso del gasto relativo en pan, pastas, cereales, papas; hortalizas, frutas, legumbres, huevos, aceites, grasas y azúcares en todos los grupos quintiles; descenso en carnes y derivados procesados y lácteos para el $\mathrm{TH}$ y QV; mantención del gasto relativo en carnes y derivados procesados, pescados y mariscos y aumento de lácteos en el QII. En bebidas y jugos procesados hay un aumento significativo del gasto; que casi se duplica para todos los grupos quintiles; de $4,0 \%$ a $8,4 \%$; en el TH y QII; y de $4,0 \%$ a $9,8 \%$ en el QV; lo que se repite en bebidas alcohólicas. El gasto en comidas preparadas y fuera del hogar, aumenta de $12,1 \%$ a $20,1 \%$; de $5,0 \%$ a $12 \%$; y de $19,1 \%$ a $30,7 \%$, para el $\mathrm{TH}$, QII y QV, respectivamente. (Figura 3). A modo de conclusión, la comparación entre el QII y el QV, permite señalar que para el QII, los grupos de alimentos más im- 
Tabla 2. Evolución del gasto relativo en alimentos del Gran Santiago. 1987 - 97 - 2007. Total Hogares y Quintiles de Ingreso II y V. En pesos (*) y porcentaje (\%)

\begin{tabular}{|c|c|c|c|c|c|c|c|c|c|}
\hline & $\begin{array}{c}\text { TH } \\
1986-87 \\
\%\end{array}$ & $\begin{array}{c}\text { TH } \\
1996-97 \\
\%\end{array}$ & $\begin{array}{c}\text { TH } \\
1996-97 \\
\%\end{array}$ & $\begin{array}{c}\text { QII } \\
\text { 1986-87 } \\
\%\end{array}$ & $\begin{array}{c}\text { QII } \\
\text { 1996-97 } \\
\%\end{array}$ & $\begin{array}{c}\text { QII } \\
\text { 2006-07 } \\
\%\end{array}$ & $\begin{array}{c}\text { QV } \\
1986-87 \\
\%\end{array}$ & $\begin{array}{c}\text { QV } \\
1996-97 \\
\%\end{array}$ & $\begin{array}{c}\text { QV } \\
\text { 2006-07 } \\
\%\end{array}$ \\
\hline Pan, pastas, cereales, papas & 22,8 & 18,7 & 18,5 & 30,4 & 22,7 & 22,4 & 15,8 & 16,9 & 13,8 \\
\hline Hortalizas frescas y procesadas & 7,7 & 7,8 & 6,8 & 8,9 & 8,9 & 8,1 & 6,7 & 8,1 & 5,5 \\
\hline Frutas frescas y procesadas & 5,4 & 5,6 & 4,8 & 5,2 & 5,5 & 4,9 & 5,9 & 7,1 & 4,5 \\
\hline Legumbres & 0,9 & 0,5 & 0,4 & 1,3 & 0,8 & 0,7 & 0,4 & 0,3 & 0,2 \\
\hline Carnes y derivados procesados & 20,4 & 18,2 & 16,7 & 19,7 & 20,4 & 19,1 & 20,6 & 18,7 & 13,2 \\
\hline Pescados y Mariscos & 1,9 & 2 & 1,9 & 1,5 & 1,7 & 1,6 & 2,2 & 2,8 & 2,1 \\
\hline Huevos & 1,9 & 1,2 & 1,2 & 2,2 & 1,5 & 1,5 & 1,6 & 1,0 & 0,8 \\
\hline Lácteos procesados & 8,2 & 7,9 & 7,9 & 6,7 & 7,8 & 7,9 & 9,5 & 10,0 & 7,8 \\
\hline Aceites y grasas & 4,6 & 3,6 & 2,6 & 5,4 & 4,1 & 3 & 3,7 & 3,6 & 2,8 \\
\hline Azúcares y dulces & 5,9 & 5 & 4,2 & 6,1 & 4,8 & 4 & 5,8 & 6,3 & 4,6 \\
\hline Bebidas y Jugos procesados & 4,0 & 9,1 & 8,4 & 4,1 & 9,6 & 9,2 & 4,0 & 9,8 & 7,0 \\
\hline Bebidas alcohólicas & 2,1 & 3,5 & 4,5 & 1,7 & 2,8 & 3,6 & 2,6 & 5,2 & 5,4 \\
\hline $\begin{array}{l}\text { Comidas preparadas y fuera } \\
\text { del hogar }\end{array}$ & 12,1 & 15 & 20,1 & 5,0 & 7,4 & 12 & 19,1 & 8,2 & 30,7 \\
\hline \multirow[t]{2}{*}{ Otros } & 2,0 & 1,9 & 1,8 & 2,0 & 2 & 2 & 2,1 & 2,1 & 1,6 \\
\hline & 100,0 & 100,0 & 100,0 & 100,0 & 100,0 & 100,0 & 100,0 & 100,0 & 100,0 \\
\hline
\end{tabular}

(*) En pesos abril de 2007. Deflactado por IPA.

Tabla 3. Evolución del gasto absoluto y relativo en alimentos procesados y naturales. Gran Santiago. 1987 - 2007. Total Hogares y Quintiles de Ingreso II y V. En pesos (*) y porcentaje (\%)

\begin{tabular}{|c|c|c|c|c|c|c|c|c|c|c|c|c|}
\hline & \multicolumn{2}{|l|}{$\begin{array}{l}\text { TH } \\
87\end{array}$} & \multicolumn{2}{|c|}{$\begin{array}{c}\text { TH } \\
2007\end{array}$} & \multicolumn{2}{|c|}{$\begin{array}{l}\text { Q11 } \\
87\end{array}$} & \multicolumn{2}{|c|}{$\begin{array}{l}\text { Q1I } \\
2007\end{array}$} & \multicolumn{2}{|l|}{$\begin{array}{l}\text { QV } \\
87\end{array}$} & \multicolumn{2}{|l|}{$\begin{array}{c}\text { QV } \\
2007\end{array}$} \\
\hline & $\$$ & $\%$ & $\$$ & $\%$ & $\$$ & $\%$ & & $\%$ & & $\%$ & & $\%$ \\
\hline $\begin{array}{l}\text { Gasto alimentos } \\
\text { procesados* }\end{array}$ & 42.457 & 38 & 90.436 & 56 & 21.843 & 31 & 59.088 & 48 & 95.762 & 51 & 180.390 & 67 \\
\hline $\begin{array}{l}\text { Gasto en alimentos } \\
\text { naturales* }\end{array}$ & 62.616 & 62 & 70.346 & 44 & 48.606 & 69 & 64.856 & 52 & 82.468 & 49 & 87.112 & 43 \\
\hline Gasto total alimentos* & 105.173 & 100 & 160.782 & 100 & 70.449 & 100 & 123.944 & 100 & 188.230 & 100 & 267.502 & 100 \\
\hline
\end{tabular}

(*) En pesos abril de 2007. Deflactado por IPA.

portantes en orden decreciente son; pan, cereales, pastas y papas; carnes; bebidas y jugos, hortalizas y lácteos, los que en total determinan casi 70\% del gasto en alimentos; para el QV, 30,7\% del gasto en comidas preparadas y fuera del hogar, más 13,8\% del grupo pan, cereales, pastas y papas y $13,2 \%$ en carnes y derivados representan casi $60 \%$ del gasto relativo, para el 2007. Destaca la baja ponderación del gasto en grupos de alimentos más protectores de la salud como pescados y mariscos, hortalizas, frutas y legumbres para ambos quintiles.
Gasto absoluto y relativo en alimentos procesados (Tabla 3)

Los alimentos procesados se seleccionaron según clasificación definida previamente. El gasto absoluto aumenta en $110 \% ; 117 \%$ y $88 \%$ para el TH; QII y QV entre 1987 y 2007. El gasto relativo, en el 2007, representa $56 \%$ para el TH; 48\% para el QII 67\% en el QV (Figura 4).

El análisis del gasto de los grupos pan y azúcares agregados de alta participación en el patrón alimentario actual, permite observar la 
sustitución de productos menos procesados por los de mayor procesamiento. Estos cambios están impactando la composición nutricional de la dieta y posiblemente, el perfil epidemiológico observado (Figura 5).

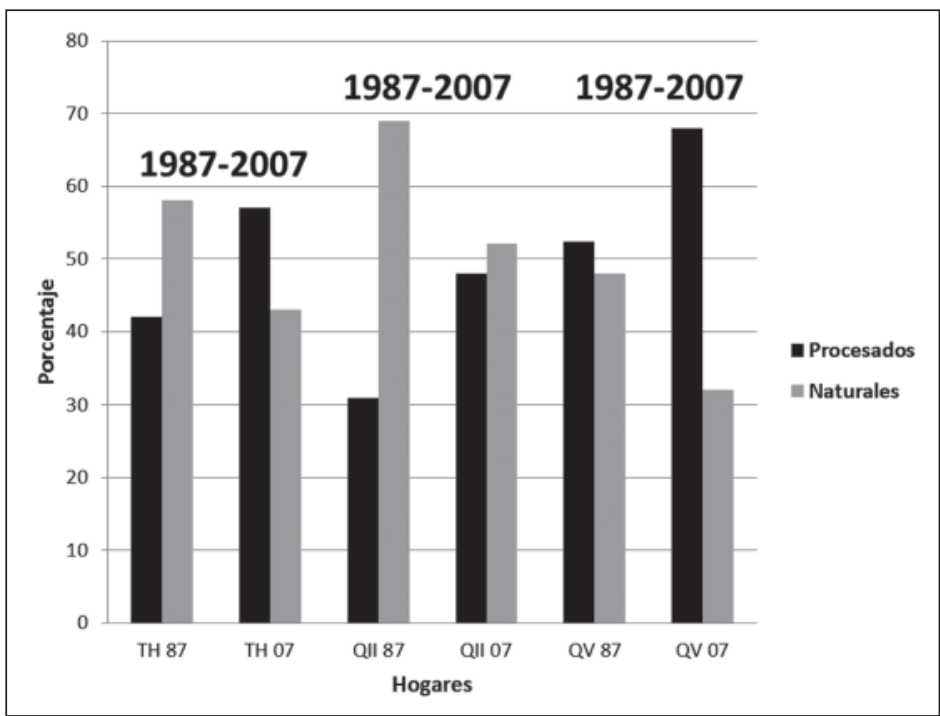

Figura 4. Gasto relativo en alimentos procesados y naturales. Total Hogares y Quintiles de Ingreso II y V. Gran Santiago*. 1987-2007. En porcentaje (\%). ${ }^{*}$ E En pesos abril de 2007. Deflactado por IPA.

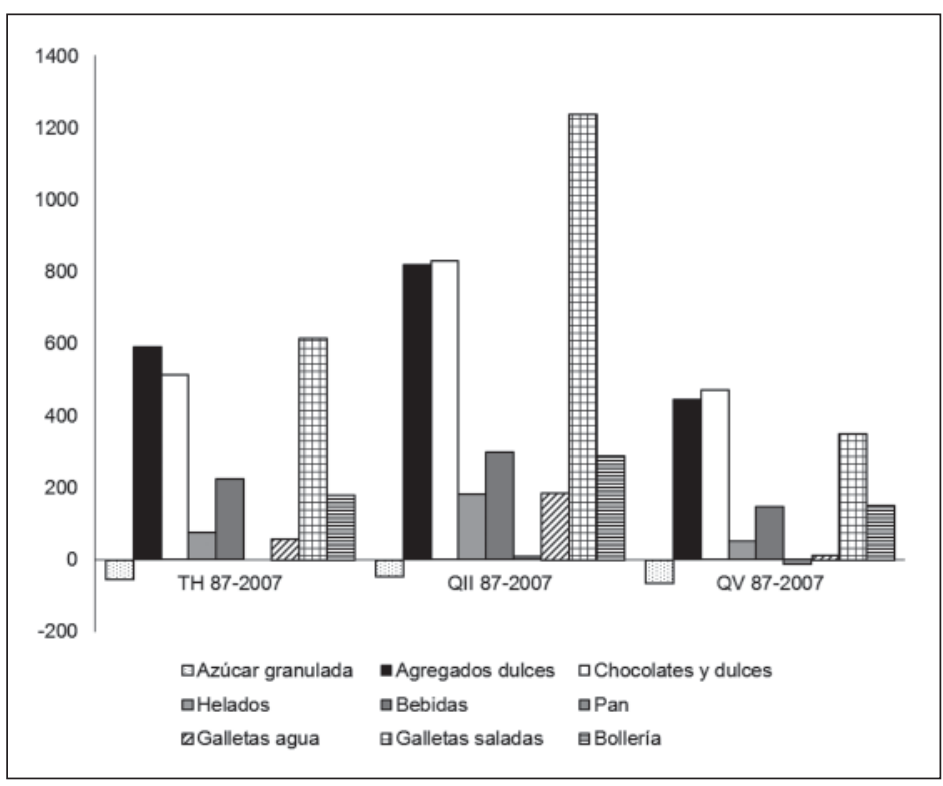

Figura 5. Cambios en el gasto absoluto de productos menos procesados por productos más procesados. Total Hogares y Quintiles de Ingreso II y V. Gran Santiago*. 1987 - 2007. En porcentaje (\%). (*) En pesos abril de 2007. Deflactado por IPA.

\section{Discusión}

En Chile, en los últimos 20 años, mejoró el poder adquisitivo, aumentó el gasto absoluto en alimentos y disminuyó su participación relativa en el gasto total, impactando el patrón y consumo alimentario de los hogares del Gran Santiago. La VI EPF muestra por primera vez, -para el TH-que el gasto relativo en Transporte y Comunicaciones $(22,3 \%)$ es mayor que el de Alimentos y Bebidas $(21,1 \%)$. El gasto en alimentos, sin distinción de ingreso, ha variado hacia un mayor gasto en productos procesados. La IV EPF (década de los 80) mostró el inicio de estos cambios, especialmente, en el QV, de mayor ingreso. En la V EPF (años 90) se aprecian para el TH y en menor magnitud para el QII; en la VI EPF (década del 2000), aumenta para todos los quintiles ${ }^{1-3}$. El cambio en la composición del gasto en alimentos, señala la disminución o desplazamiento de productos que eran parte de una alimentación más tradicional y más saludable, de menor densidad energética, grasas totales y saturadas, azúcares agregados, sal, mayor contenido de fibra y micronutrientes hacia alimentos procesados, productos de alta densidad energética, mayor contenido en grasas totales y saturadas, azúcares agregados, sodio, bajos en micronutrientes y fibra dietaria ${ }^{18,19}$. Este proceso se viene observando desde mediados de los 80 , coincidente con la globalización y la irrupción de las trasnacionales de la industria alimentaria en países de menor desarrollo ${ }^{9,10}$. Se grafica esta situación en el aumento del consumo y predominio de las bebidas azucaradas en los hogares, por sobre la condición económica. Asimismo, 
se observa un alejamiento paulatino y silencioso de las dietas tradicionales hacia una dieta global, con una gran cantidad de alimentos procesados, de origen animal, azúcares agregados, grasas saturadas y trans, y el incremento del alcohol ${ }^{9,19-22}$. Este patrón alimentario puede estar contribuyendo al aumento de enfermedades crónicas relacionadas con la dieta, entre ellas, la obesidad. Lo más grave, es que la capacidad de respuesta y reacción de la comunidad científica ha estado desfasada y ha avalado el desarrollo e incorporación de productos sustitutos de las dietas tradicionales ${ }^{23,24}$.

En Chile, en los 80, inicio de estos cambios, la comunidad científica se sorprendió con los estudios de Berríos et al cuya evidencia más significativa se encontró a fines de los $90^{25-27}$. Las ECV 2000 y 2003, los estudios de los Años de Vida Ajustados por Discapacidad (AVISA) en 1997 y 2008 14,16,29-30; la ENSA del año 2006 y la del 2009-2010, han hecho evidente los cambios epidemiológicos de la población ${ }^{15,16}$. La ENSA 2009-2010, señala que los mayores de 18 años tienen un consumo de 9,8 g de sal, casi el doble de lo recomendado por la OMS; $15,7 \%$ consume 5 o más porciones de verduras y frutas al día y alerta del consumo de 55,6 g de alcohol día, lo que concuerda con el aumento en el gasto en alcohol observado en la VI EPF ${ }^{13,16}$.

En 1987, 7\% de los escolares de primer año básico tenía obesidad, en contraste de $21 \%$ que la presenta en el $2010^{32}$. La obesidad infantil se asocia a una mayor probabilidad de muerte prematura, discapacidad y riesgo de diabetes en la adultez. Según la OMS, en el 2015, el sobrepeso y la obesidad afectarán a 2.300 y 700 millones de adultos, respectivamente ${ }^{33,34}$.

El patrón alimentario actual y sus cambios, se aleja significativamente de todas las recomendaciones emanadas de organismos rectores en alimentación y nutrición ${ }^{33,34}$. Las recomendaciones de la guía alimentaria publicada por el Departamento de Agricultura de Estados Unidos de Norteamérica (USDA) y el Departamento de Salud y Servicios Humanos (HHS) pueden extrapolarse a nuestra realidad ${ }^{35}$. El aumento en el gasto en alimentos procesados indica que en los hogares analizados, i) los productos con preservantes, grasas sólidas y azúcares, han aumentado entre 1987 y 2007 y que, en la mayoría son altos en sodio; ii) Respecto a las calorías grasas, un estudio previo de los autores señaló que el aporte calórico de grasas saturadas, era mayor al 10\% recomendado, lo que puede extrapolarse a esta investigación, dado que el patrón de gasto en alimentos entre 1997 y 2007, no experimenta cambios significativos, sino por el contrario lo consolida ${ }^{6}$; iii) En el mismo estudio, respecto a los azúcares simples, los hogares superaron entre dos y tres veces el máximo del 10\% de porcentaje de calorías provenientes de éstos, recomendadas por FAO/OMS 20037. En relación al aumento en pescados, verduras, frutas, granos y legumbres nos indica que estas recomendaciones no se logran, como tampoco la diversificación de las fuentes proteicas.

Los cambios del gasto en alimentos, el peso de los productos procesados, la reafirmación del patrón de consumo en estos últimos 20 años, amerita un análisis de los factores que están interactuando en la adopción de la dieta "moderna asociada al avance tecnológico" y su génesis en las enfermedades nutricionales. En esta mirada, investigadores sugieren repensar los conceptos tradicionales en alimentación y nutrición, analizar la clasificación y evaluación de la composición nutricional de los alimentos procesados, hacerse cargo de este patrón de consumo, su impacto en la salud y orientar consumos recomendados en estos alimentos. ¿Será momento de exigir un compromiso social de verdad a las industrias y empresas de alimentos? ¿El Estado será capaz de dar y aplicar los marcos regulatorios que se requieren para fijar los límites? ¿Y la sociedad, se hará responsable, del autocuidado de su salud?

\section{Referencias}

1. Instituto Nacional de Estadísticas. Estructura del gasto de los hogares del Gran Santiago por grupo quintil de hogares. En: IV Encuesta de presupuestos familiares 1987-1988, v.3. Santiago, Chile: INE, 1989.

2. Instituto Nacional de Estadísticas. Estructura del gasto de los hogares del Gran Santiago por grupo quintil de hogares. En: V Encuesta de presupuestos familiares 1996-1997. v.3 Santiago, Chile: INE, 1999.

3. Instituto Nacional de Estadísticas. Estructura del gasto de los hogares del Gran Santiago por grupo quintil de hogares. En: VI Encuesta de presupuestos familiares 2006-2007. Santiago, Chile: INE, 2009.

4. Crovetto M. Cambios en la estructura alimentaria y consumo aparente de nutrientes de los hogares del Gran Santiago 1988-1997, Rev Chil Nutr 2002; 29: 24-32.

5. Crovetto M, Uauy R. Cambios en la disponibilidad de 
alimentos en el Gran Santiago por quintiles de ingreso.1988-1997. Arch Latinoam Nutr 2008; 58: 40-8.

6. Crovetto M, Uauy R. Cambios en el consumo aparente de nutrientes en el Gran Santiago 1988-1997 en hogares según ingreso y su probable relación con patrón de enfermedades crónicas no trasmisibles. Rev Med Chile 2010; 138: 1091-108.

7. Organización Mundial de la Salud (OMS), Organización de las Naciones Unidas para la Agricultura y la Alimentación (FAO). Dieta, nutrición y prevención de enfermedades crónicas. En: Informe de una Consulta Mixta de Expertos. Serie de Informes Técnicos, 916. Ginebra, 2003.

8. Ludwing D. Technology, Diet, and the Burden of Chronic Disease. Am Med Assoc JAMA 2011; 13: 1352-3.

9. Monteiro C. The big issue is ultraprocessing, World Nutrition 2010; 6: 237-69.

10. Monteiro C. FS Gomes, Cannon G. El ataque de aperitivos. Rev Panam Salud Públ 2010; 100: 975-81.

11. Instituto Nacional de Estadísticas (INE). Índice de precios al consumidor (IPC). Serie 1986-1999. Santiago: INE, 1999.

12. Instituto Nacional de Estadísticas (INE). Índice de precios al consumidor (IPC) base diciembre 1998: Aspectos metodológicos. Santiago: INE, 1999.

13. Instituto Nacional de Estadísticas (INE). Índice de precios al consumidor (IPC) base diciembre 2009: Aspectos metodológicos. Santiago: INE, 2009.

14. Ministerio de Salud (MINSAL). I Encuesta Nacional de Calidad de Vida y Salud. Santiago: MINSAL, 2000.

15. Ministerio de Salud (MINSAL). Encuesta Nacional de Salud: Informe final. Santiago: MINSAL, 2003.

16. Ministerio de Salud (MINSAL). II Encuesta de Calidad de Vida y Salud. Santiago: Departamento de Epidemiología y Departamento de Promoción de Salud, MINSAL, 2006.

17. Ministerio de Salud (MINSAL). Encuesta Nacional de Salud: Informe preliminar. Santiago: MINSAL, 20092010.

18. Uauy R, Monteiro CA. The challenge of improving food and nutrition in Latin America. Food Nutr Bull 2004; 25: $175-82$.

19. Prentice AM, Jebb SA. Fast foods, energy density and obesity: a possible mechanistic link. Obesity Reviews 2003; 4: 187-94.

20. Uauy R, Vio F. Health and nutrition transition in developing countries: the case of Chile. En: The Nation's Nutrition. International Life Sciences Institute (ILSI), Washington, D. C. USA 2007; p. 117-28.

21. Uauy R, Puska P. Health significance of fat quality of the diet. Expert Meeting (Barcelona, 1-2 February 2009).
Basel (Switzerland): S. Karger AG., 2009

22. Organización Panamericana de la Salud (PAHO), Organización Mundial de la Salud (OMS). Las Américas libres de grasas trans: conclusiones y recomendaciones. Washington, D.C. USA, OPS/OMS, 2007.

23. Organización Panamericana de la Salud (OPS). Alcohol y Salud Pública en las Américas: Un caso para la acción [en línea]. 2007. <http://www.paho.org/Spanish/DD/ PIN/A\&SP>

24. The Lancet. From what we will die in 2020. The Lancet 1997; 349 (9061): 1263.

25. Berríos X. Tendencia Temporal de los factores de riesgo de enfermedades crónicas: ¿la antesala silenciosa de la epidemia que viene? Rev Med Chile 1997; 11: 1405-7.

26. Berríos $\mathrm{X}$, et al. Prevalencia de factores de riesgo de enfermedades crónicas. Estudio en población general de la Región Metropolitana, 1986-1987. Rev Med Chile 1990; 118: 597-604.

27. Koch E, Silva C, Manríquez L, Ahumada C. Proyecto San Francisco, parte I: alta prevalencia de factores de riesgo cardiovascular en población adulta mayor de 15 años. Rev Chil Card 2000; 19: 27-42.

28. Koch E, Otárola A, Manríquez L, Kirschbaum A, Paredes M, Silva C. Predictores de eventos cardiovasculares no fatales en una comunidad urbana en Chile: experiencia de seguimiento Proyecto San Francisco. Rev Med Chil 2005; 133: 1002-1012

29. Ministerio de Salud (MINSAL). Informe Final, Estudio de prioridades de inversión en Salud Estudio Carga de Enfermedad. Santiago, Chile. MINSAL, 1996.

30. Ministerio de Salud (MINSAL). Informe final: Estudio de carga de enfermedad y carga atribuible. Santiago, Chile: MINSAL, 2008.

31. Junta Nacional de Auxilio Escolar y Becas (JUNAEB). Situación nutricional de los escolares chilenos [en línea]. $<$ http://ins.junaeb.cl $>$

32. Ministerio de Salud (MINSAL). Sistema de vigilancia nutricional”. [en línea]. <http//:www.minsal.cl>

33. Organización Mundial de la Salud (OMS). Estrategia Mundial sobre régimen alimentario, actividad física y salud. En: 57a Asamblea Mundial de la Salud. Organización Mundial de la Salud. Ginebra, Suiza; OMS, 2004.

34. United Nations Standing Committee on Nutrition. Progress in Nutrition. Sixth Report on the World Nutrition Situation. Geneva, Switzerland, UNSCN, 2010.

35. United States Department of Agriculture Center for Nutrition Policy and Promotions. Dietary Guidelines for Americans, "Report of the Dietary guidelines Advisory Committee on the Dietary Guidelines for Americans" [en línea]. 2010. http://www.cnpp.usda.gov/Dietary Guidelines.htm 